\title{
THE FORMATION OF MULTIPLE SYSTEMS BY DYNAMICAL INTERACTION IN CLUSTERS
}

(Invited Paper)

\author{
S. J. Aarseth \\ Institute of Astronomy \\ University of Cambridge
}

\begin{abstract}
RESUMEN
Esta reseña se dedica especialmente a la discusión de la formación y evolución de binarias en sistemas estelares obtenida con técnicas de $\mathrm{N}$ cuerpos. Los mecanismos más sencillos de formación consisten en la eyección de sistemas triples ligados y la captura resultante de encuentros hiperbólicos triples. Sin embargo, no se puede explicar de esta manera el gran número de binarias astrofísicamente cercanas a menos que las estrellas se formen en grupos compactos. Cálculos numéricos muestran que la evolución de cúmulos, con varios centenares de estrellas, es dominada invariablemente por una binaria, central que absorbe una fracción substancial de la energía total. Se sugiere que una binaria visual en el centro del cúmulo de las Yadas puede estar relacionada a este proceso.
\end{abstract}

\begin{abstract}
ABSTRAGT
This review is mainly devoted to a discussion of binary formation and evolution in stellar systems, as described by $\mathrm{N}$-body techniques. The simplest formation mechanisms consist of ejection from bound triple systems and capture arising from hyperbolic three-body encounters. However, the large number of astrophysically close binaries cannot be accounted for in this way unless most stars are formed in compact groups. Numerical calculations show that the evolution of clusters containing several hundred members is invariably dominated by one central binary which absorbs a large fraction of the total energy. It is suggested that a visual binary in the core of the Hyades cluster may have been associated with this process.
\end{abstract}

\section{INTRODUGTION}

In this review we consider the dynamical origin of bound sub-systems in different types of stellar systems. Because of the complexities involved, this problem has mainly been studied by numerical N-body techniques in which the individual particle orbits are calculated as accurately as possible. Although such simulations usually adopt idealized assumptions, a variety of initial conditions have been explored and the results permit certain general conclusions to be drawn.

The present discussion concentrates mainly on binaries which form the most numerous sub-group among the multiple systems. At the outset it should be emphasized that even these simple systems may not have a common mode of origin. Three main formation mechanisms have been considered: fission, multiple condensations, and many-body encounters (also referred to as capture). The first process is associated with physical instabilities rather than dynamical interactions and will therefore not be discussed further; however, it represents an attractive alternative for the origin of very close binaries.

According to the fragmentation theory of star formation, compact sub-groups may condense within clusters or associations. Unless already stable, such systems would eject their members rapidly by close encounters until only one binary remained, the final semi-major axis being somewhat smaller than the initial radius. An additional consequence of this model is that the escape velocities could be large, 
thereby providing a possible explanation for the runaway stars.

The third mechanism of binary formation is based on three or more strongly interacting stars producing a bound pair by capture. If can be shown that the probablity of suitable triple encounters is extremely small at typical interstellar densities. Conditions near cluster centres are most favourable, but cven so the yield of cloee binaries is likely to be small in normal circumstances. However, wide binaries can be formed more readi'y and their relative orbits may shrink by subsequent perturbations. A complete understanding of the binary phenomenon therefore requires additional information about the evolutionary effects of passing stars. This problem is treated theoretica!ly elsewhere in this volume for galactic binaries (Heggie 1977, King 1977), whereas a combination of theory and numerical results have clarified the situation in the more difficuli case of cluster binaries (Heggie 1975, van Albada 1968a, Aarseth 1971).

In the following section we first consider the simplest type of binary formation which occurs in strongly bound triple systems when one of the particles escapes. Numerical experiments on triple systems with positive energy are also discussed; in this case the pa: ticle motions are assumed to be representative of large clusters whose evolution cannot be simulated by direct methods. Quite a few small groups and medium-s'ze c'uster models have been studied by now. In Sections 3 and 4 we summarize numerical results relevant to the binary phenomenon and also add some simple theoretical considerations. The final section contains a brief discussion of the astronomical implications, together with recent data on binaries in the Hyades.

\section{THREE-BODY SYSTEMS}

A number of investigations have been devoted to the study of bound triple systems (Agekyan and Anosova 1968a, b, Standish 1972, Szebehely 1972, Valtonen 1974), and a general review can be found elsewhere in this volume (Szebehely 1977). Such calculations give detailed information about the final binary configurations as well as the time-scale of disruption. The results may also be applicable to clusters or associations if it is assumed that stars form in small groups which are sufficiently compact to be consideret in isolation.

The escape of one particle incvitably leaves behind a bound pair whose energy exceeds the total ere-gy in absolute value. Although this cxcess may be arbitrarily large for close triple encounters, such cxamples are rare statistically even when permitted (i.e., zero angular momentum). Thus in one series of 115 exper:ments with the particles initially at resi (Szebehely 1972), the most energetic binary cxceeded the total energy by a factor of 8 but only a few cthers containel twice the total encrgy. W'c may conjecture that the average energy excess should become smaller with increasing angular momentum, since such systems also have less critical triple cncounters.

Different studies confirm the expectation that the escaping farticlc is usually the lightest. Even so, there is a significant probability $(\simeq 10 \%)$ for either of the two other particles to be ejected, at least for moderate mass dispersion (Szebehely 1972). Another characteristic feature is that a mass spectrum reduces the average lifetime by a factor of 3 with respect to similar equal-mass systems which disintegrate in about 30 mean crossing times (Anosova 1969, Standish 1972). Most three-body calculations have been carried out in two dimensions. It is fortuitous that the extension to three dimensions does not affect the results significantly (Valtonen 1974).

If a given class of binaries originated in bound triple systems, it may be possible to obtain further information about the initial conditions by analyzing the eccentricity distribution. For example, it has been noted that the final eccentricities tend to decrease when the angular momentum is increased (Standish 1972, Valtonen 1974). Furthermore, such an hypothesis should have observational consequences for the distribution of mass ratios in binaries.

After this brief discussion of bound triples we now turn to the case when the thrce particles approach each other along hyperbolic orbits. The frequency of such encounters increases as the density cubed, whereas the probability of forming a bound pair of fixed energy is smaller for large velocity dispersions. We may therefore expect this process to be most efficient in the central regions 
of star clusters. Since the dynamics of globular clusters cannot be simulated by N-body methods, three interacting stars may be considered as an isolated system, provided that their mutual distances are sufficiently small. This problem was first studied by Agekyan and Anosova (1971), and their method of sampling initial conditions was adopted in a more extensive investigation performed recently (Aarseth and Heggie 1976).

The basic idea is to calculate a large number of three-body interactions where the initial orbits intersect a sphere of radius $r_{0}$. Each set of experiments is characterized by a fixed value of the dimensionless parameter

$$
\mathrm{X}=\mathrm{r}_{0} \overline{\mathrm{V}}^{2} / 2 \mathrm{Gm} .
$$

The initial velocities are sampled from a Maxwellian with mean square value $\overrightarrow{\mathrm{V}}^{2}$ and we choose particles of equal mass $\mathrm{m}$. Having selected appropriately randomized coordinates, the integration is started with the particles well outside the sphere of maximum interaction. Each calculation is terminated when one of the pair separations exceeds the maximum initial separation, whereupon the condition for bound two-body motion is tested.

A comparison shows that the probability of binary formation, $\mathrm{P}$, is significantly greater than obtained by Agekyan and Anosova (factor of 9 for $X=5$ / $\sqrt{3})$. For large values of $X(16 \leq X \leq 1024)$ the present results are well approximated by $P \simeq 4 \mathrm{X}^{-2}$, in satisfactory agreement with a theoretical derivation which gives $\mathrm{P} \simeq 7 \mathrm{X}^{-2}$ for $\mathrm{X} \gg 1$. Saturation effects become increasingly important at small $\mathrm{X}$ values but, even so, $\mathrm{P} \simeq 0.5$ for $\mathrm{X}=1$.

The numerical results may be applied to real clusters by relating $r_{0}$ to the mean particle separation $\mathrm{d}=\mathrm{R} \mathrm{N}^{-1 / 3}$, where $\mathrm{R}$ is the cluster radius and $\mathrm{N}$ is the total number of particles. Assuming constant density, the total number of binaries formed by three-body encounters within $r_{0}=d$ during the time $t$ is estimated to be

$$
n_{b} \simeq \frac{1}{4} \mathrm{P} \mathrm{N}^{4 / 3}(\overline{\mathrm{R}} / \mathrm{R}) \mathrm{t} / \mathrm{t}_{\mathrm{cr}},
$$

where $\bar{R}$ is the virial theorema scale factor and $t_{c r}$ is the crossing time defined by $t_{c r}=2 \overline{\mathrm{R}} / \overline{\mathrm{V}}$. The empirical result $P \simeq 4 \mathrm{X}^{-2}$ is now substituted and together with $X=\frac{1}{4} N^{3 / 3} R / \bar{R}$ obtained from the virial theorem, Equation (2) simplifies to

$$
n_{b} \simeq 16(\bar{R} / R)^{3} t / t_{c r} .
$$

We note that the independence of $N$ is due to the relation $\mathrm{P} \propto \mathrm{X}^{-2}$. Equation (3) also applies to smaller volumes, except that the numerical constant should be reduced by the volume factor.

Real clusters do not have constant densities and the estimate above is therefore somewhat uncertain. Even so, the fraction of dynamically formed binaries, $\mathrm{n}_{\mathrm{b}} / \mathrm{N}$, is likely to be very small in most clusters. Furthermore, typical binding cnergies would be smaller than the mean kinetic energy of single stars by a factor $\mathrm{X}$. Such binaries would therefore tend to be disrupted by subsequent interactions, as discussed elsewhere in this volume (Heggie 1977, King 1977). However, relatively stable binaries may still from in the contracting cores of globular clusters and be ejected into regions of lower density.

\section{SMALL GROUPS}

The behaviour of N-body systems is qualitatively similar for a wide range of particle numbers and the division of this subject into two sections is merely done for astrophysical convenience. From the preceding discussion it may appear that the efficiency of binary formation by multiple encounters is too small to be significant in these systems. As an example, the parameters $N=100, R \simeq 2 \bar{R}$ would correspond to $\mathrm{X} \simeq 11$ for triple encounters within the mean particle separation. Consequently, the probability of forming a wide binary would be a few per cent for each interaction of this type and the capture probability would be enhanced further at smaller particle numbers or more energetic encounters.

Analysis of the numerical results shows that binaries are more important in systems with unequal masses where there is a preference for heavy particles to combine. This feature can be understood in terms of two separate effects. In the first place, the theoretical cross-section for binary formation contains approximately the $5 / 2$ power of the mass of both components (Heggie 1975), and an alter- 
native cxpression based on phase-space arguments also shows a strong mass dependence (Miller 1975). Moreover, the numerical models exhibit a pronounced mass segregation, with the heavy particles concentrated towards the centre. A significant mass segregation is usually achieved after the first few crossing times. At this stage some of the massive particles form a strongly bound nucleus, whereas other particles either escape or are ejected into low density regions. The rapid evolution of the nucleus is terminated by the formation of a close binary which continues to shrink by further encounters. Systematic studies with $\mathrm{N}=10$ particles illustrate these general trends (van Albada 1968a). In nearly all these examples, the binding energy of the final binary exceeds the total energy in absolute value. Furthermore, only one out of 22 unequal mass binaries does not contain either of the two heaviest particles, whereas about half have the maximum mass. Escaping binaries which tend to be wide are not uncommon; i.e., there are 12 such pairs among 151 cscapers.

As the central binary cvolves, it presents a reduced target for close cncounters. In due course such a composite particle may become the component of a new binary. This behaviour was first noted by van Albada (1968a) who also emphasized the apparent stability of triple systems with hierarchical structure. Subsequent investigations (Harrington 1972) have demonstrated that the ratio of the outer pericentre to the inner semi-major axis determines the stability of hierarchical configurations, with different critical values for direct and retrograde motion. We note, however, that these conclusions are based on numerical calculations, whereas statements of absolute stability require mathematical proof which is not yet available. Even so, the numerical approach may provide useful information for times of astronomical interest.

From the preceding discussion it follows that the angular momentum is an important stability parameter for triple systems. Similar considerations apply to hicrarchical systems of greater multiplicity; for cxample, most spectroscopic binaries are dynamically cquivalent to single stars of the corresponding mass. Although the stability of such multiple systems can be understood, their origin is still uncertain. It may be possible to distinguish between two main ideas, i.e., direct formation with favourable initial conditions or evolution from unstable systems. Recent calculations (Harrington 1974) show that systems with four particles generated at random in two and three dimensions give rise to a significant proportion of fairly stable triples. As regards further work, it would be especially interesting to see whether some of the angular momentum associated with the differential galactic rotation can be retained by a small number of bound stars. However, the total number of multiple systems may well be too large to be consistent with dynamical evolution from typical clusters rather than small groups.

On the observational side, the binary phenomenon is specially prominent among the bright stars in trapezia and associations. A dynamical model has been proposed which would link the runaway stars with close binaries in compact groups containing five or six stars (Poveda, Ruiz and Allen 1976). A characteristic feature of these models is that the particles are distributed in almost symmetrical configurations and with small initial velocities. The subsequent contraction produces strong interactions, with preferential accelerations giving rise to one or two energetic escapers. As a consequence of energy conservation, such events also lead to the formation of close binaries. This behaviour is consistent with theoretical expectations for the general three-body problem. Thus if $\mathrm{X} \ll 1$ [cf. Equation (1)] binary formation is inevitable, giving a semi-major axis comparable to one third of the minimum perimeter. Likewise, the product $\mathrm{v}^{2} \mathrm{a}$ (escape velocity squared times the semi-major axis) should approach an asymptotic value (Szebehely 1974). As far as the observational predictions are concerned, it remains to be seen whether the assumption of an initial collapse can be justified.

Another astrophysically inspired simulation is based on models of trapezium systems (Allen and Poveda 1974). The initial parameters of six representative stars are inferred from observations of the Orion Trapezium, indicating approximate equilibrium. After the first 30 crossing times, about twothirds of the systems still conform to the traditional definition of trapezia. From the observational data, this time-scale corresponds to about $10^{\circ} \mathrm{yrs}$. The dynamical lifetimes of trapezia containing $O$ stars are therefore not much smaller than their maximum 
ages inferred from stellar evolution, whereas previous discussions of these multiple systems either assumed expansion or smaller ages. This study gives additional information about dynamical binaries. Thus in 21 out of 30 examples, the two heaviest particles form the closest pair. As was noted previously (van Albada 1968a), the eccentricity distribution follows approximately the law $f(e)=2 e$ predicted for binaries in statistical equilibrium (cf. Heggie 1975).

\section{LARGE N-BODY SYSTEMS}

Most open stars clusters contain at least a few hundred members and such systems therefore pose a natural target for $\mathrm{N}$-body simulations. This subject has been reviewed elsewhere recently (Aarseth 1973, Wielen 1974), hence we need only emphasize aspects directly relevant to multiple systems. So far, the main computational effort has been directed to the study of simple isolated systems but some results are also available for more realistic models.

When discussing cluster binaries it is of interest to compare the binding energy, $\mathrm{E}_{\mathrm{b}}$, to the mean kinetic energy of field particles as well as to the total energy, E. Using dimensionless units, the respective quantities are defined by $\varepsilon=\left|\mathrm{E}_{\mathrm{b}}\right| / \frac{1}{2} \overline{\mathrm{m}} \overline{\mathrm{V}}^{2}$ and $\lambda=\left|E_{b} / E\right|$. Binaries which cannot be disrupted by particles of the mean kinetic energy may be said to be close, corresponding to $\varepsilon \geqslant 1$. It is characteristic of the large $\mathrm{N}$-body systems that at least one such binary forms at the cluster centre. Typical timescales for $N=250$ range from $t / t_{\mathrm{cr}} \simeq 40$ for equal masses to $t / t_{\mathrm{cr}} \simeq 5$ for a smooth spectrum (Aarseth 1971). Both capture and exchange play an important role during the subsequent evolution which proceeds in the direction of increased mass (where appropriate) and energy. The devolpment towards more energetic configurations is quite rapid at first but a gradual slowing-down occurs as the nucleus is depleted of particles. Thus in one model with $\mathrm{N}=500$ and a maximum mass ratio of 32 , the central binary accounts for more than 50 per cent of the total encrgy after only 12 crossing times. It is significant that the final binary consists of the first and third most massive particle; similar cnergetically favourable configurations are also reached in other models. The changes in mass and energy are to some extent related and the systematic bchaviour can be understood from simple considerations (Aarseth 1971).

Recent theoretical work (Heggic 1975) has clarified many aspects of the binary problem. This treatment distinguishes between 'soft' and 'hard' binaries defined by $\varepsilon<1$ and $\varepsilon>1$, respectively. The soft binaries are characterized by short lifetimes but a small proportion may become more stable by 'cascades'. Hard binaries, on the other hand, tend to become more energetic by close encounters, in good agreement with the numerical calculations. This 'watershed' effect has been confirmed by three-body experiments (Hills 1975).

The parameter $\lambda$ defined above may be interpreted as an evolution measure. If only the bound particles are included in the calculation of the total energy, the limiting case of one remaining binary would correspond to $\lambda=1$. Since the proportion of escaping particles is small in most calculations, energy conservation requires that a significant increase in $\lambda$ is matched by a corresponding cluster expansion. As the contrast between the central binary and the nucleus increases conditions become favourable for the formation of fairly stable hierarchical configurations. The number of such examples is sufficiently large to be taken as a general trend (Aarseth 1974), thus confirming the similarity with much smaller systems. A study of the stability of hierarchical systems reveals some interesting aspects. The usual phenomena of capture and exchange take place, gradually increasing the binding energy of the outer component until disruption cannot be caused by single field particles. As the outer orbit shrinks further, however, the pericentre distance may no longer satisfy the condition for internal stability (cf. Harrington 1972). It may be noted that the stability criterion $\varepsilon>1$ does not apply to encounters with the inner binary components, whose mean relative velocity cxceeds the rms velocity by a factor $\varepsilon^{1 / 2}$.

A similar type of internal disruption occurs if the cccentricity of the outer orbit reaches a critical valuc. This mode of disruption is likely to be more frequent, since the time-scale for significant changes in the eccentricity is shorter than for corresponding changes in the energy (Heggie 1975). In either 
case such strong interactions usually lead to one of the particles being ejected with high velocity, whereas the surviving binary becomes even more energetic. Un'ess the recoil effect is very large and results in esaape or ejection into the halo, the process. can start all over again with the same binary; alternatively, another phase of core contraction may follow.

The spherical systems which have been discussed so far are somewhat idealized and it is desirable to explore a wider range of initial conditions. One such attempt (Aarseth and Hills 1972) was guided by the fragmentation theory of star formation which predicts an initial hierarchy of collapsing sub-clusters. The evolution of each sub-cluster first led to the formation of a close binary, some of which were destroyed in subsequent collisions. Nevertheless, the decreasing population of surviving binaries dominated the cluster evolution to a greater extent than in the simpler systems, reaching $\lambda \simeq 0.90$ after only a few crossing times. Following a period of decreased dynamical activity, the calculation was simplified using the centre of mass approximation for three extremely close pairs. Most of the remaining binaries were not significantly affected by perturbations, hence the formation of a long lived binary consisting of previously combined components could still represent a fairly stable quadruple system.

Another possibility is that binaries are present initially. Preliminary calculations (Aarseth 1975) indicate that a modest initial population of close binaries $(\varepsilon=10)$ may eventually dominate the central cluster region if their combined mass exceeds the average mass. These binaries are important in preventing the nucleus from attaining high densities, i.e., the close binaries themselves absorb an increasing amount of binding energy. Strong interactions between such energetic binaries may lead to disruption or ejection, in both cases leaving behind a more loosely bound cluster. We may therefore conjecture that the conditions would eventually become favourable for two close binaries to com. bine into a stable quadruple system.

Among the additional effects which influence the evolution of open clusters, the galactic tidal field and mass loss from stars have been included in the simulations. In the first place, the binary phenomenon itself is not significantly affected by the tidal field, which merely tends to reduce the cluster life- times (Hayli 1971, Wielen 1974). On the other hand, the assumption of mass loss by supernova events may affect dynamically formed binaries if the most massive particle is among the components (Aarseth 1973). The question of whether mass loss occurs before the most massive cluster member becomes a binary component depends on the adopted parameters which are uncertain. Only three examples of mass loss involving close binaries were noted and the new configurations remained bound but with increased periods.

At the present time the precise conditions for mass loss are subject to debate and the realistic simulations may need to be modified. However, most open clusters are sufficiently young to contain at least moderately massive stars which may still give rise to significant binary activity.

\section{OBSERVATIONAL IMPLICATIONS}

The numerical calculations reveal several binary processes which may be of astrophysical relevance. In the first place, there is a strong preference for dynamically formed binaries to contain massive components even though the number of such particles is usually quite small. Binaries which are not massive initially tend to evolve towards the maximum mass configuration either by capture followed by ejection of the lightest particle, or by exchange. If the particle masses are equal, the exchange process appears to be more effective in transforming the binary identity. Unless formed recently, any cluster binary with a dynamical origin is therefore likely to have replaced one or both of its original com. ponents, and likewise for initial binaries in the appropriate energy range.

Although some of the binaries formed dynamically become very energetic, the corresponding semi-major axis would still be relatively large when expressed in physical units appropriate to open clusters. Using the previously defined evolution measure $\lambda$ for a binary of mass components $m_{1}$ and $m_{2}$, we obtain the semi-major axis

$$
\mathbf{a}=\frac{2}{\lambda \mathrm{N}^{2}}\left(-\frac{\mathrm{m}_{1} \mathrm{~m}_{2}}{\overline{\overline{\mathrm{m}}}^{2}}\right) \overline{\mathrm{R}}
$$

where no distinction is made between the initial and final total energy of the bound cluster. To quote 
one extreme example of a small semi-major axis, $\lambda=0.20$ for an equal-mass model with $\mathrm{N}=250$ particles (Aarseth 1974). Adopting a virial theorem scale factor $\bar{R} \simeq 2 \times 10^{5}$ AU would then give $\mathrm{a} \simeq 30 \mathrm{AU}$ for the closest binary in this calculation, still much wider than typical spectroscopic binaries.

Apart from energy considerations which require that $\lambda<1$, the dynamical evolution of a cluster binary may be restricted further by recoil effects. It has been noted that close binaries tend to change their binding energy by discrete jumps rather than by slow diffusion (Aarseth 1971), and this behaviour can also be understood using certain theoretical results (Heggie 1975). Using conservation of linear momentum, the associated recoil kinetic energy due to an interaction with the mass-point $m_{3}$ can be written as

$$
\mathrm{T}_{\mathrm{r}}=\left(\mathrm{m}_{1}+\mathrm{m}_{2}\right)\left(\mathrm{m}_{1}+\mathrm{m}_{2}+\mathrm{m}_{3}\right) v_{\mathrm{cm}}^{2} / 2 \mathrm{~m}_{3},
$$

where $v_{\mathrm{cm}}$ is the centre of mass velocity of the binary. A binary would then be in danger of ejection from the cluster if its semi-major axis approaches the critical value

$$
\mathrm{a}_{\mathrm{crlt}} \simeq \frac{\mathrm{Gm}_{1} \mathrm{~m}_{2} \mathrm{~m}_{3}}{\left(\mathrm{~m}_{1}+\mathrm{m}_{2}\right)\left(\mathrm{m}_{1}+\mathrm{m}_{2}+\mathrm{m}_{3}\right) v_{\mathrm{o}}{ }^{2}},
$$

where $v_{e}$ is the escape velocity. As an upper limit we have assumed an energy change $T_{r}$ equal to the binding energy itself. Adopting $v_{\mathrm{e}}=2.6 \mathrm{~V}$ for the central escape velocity in a Plummer model, Equation (6) gives $\mathrm{a}_{\text {crit }} \simeq 0.05 \overline{\mathrm{R}} / \mathrm{N}$ for an equal-mass system, comparable to the example quoted above. Equations (4) and (6) indicate that two massive binary components permit a greater proportion of the total energy to be absorbed before recoil effects become important.

The present derivation excludes the important case of a heavy particle $m_{3}$ being exchanged with one of the lighter binary components after a close encounter. Comparison with a recent treatment (Hills 1976) shows that Equation (6) is still approximately valid for a modest mass range, although a slightly smaller semi-major axis is permitted.

From the preceding discussion it follows that most cluster binaries of dynamical origin may be relatively wide and therefore difficult to detect ob- servationally. A recent search for binaries in the Hyades cluster (Oort 1975), indicates that seven visual binaries are probable members, five of which have $\mathrm{a}<1^{\prime \prime}$. The cluster has a pronounced core of eight bright stars within a radius of $0.35 \mathrm{pc}$ and at least six of these stars are either known or suspected spectroscopic binaries (Pels, Oort and PelsKluyver 1975). One of the central stars, $80 \mathrm{Tau}$, is a visual binary with the following parameters (Aitken 1932) : $\mathrm{a}=1.04, \mathrm{e}=0.79, \mathrm{~m}_{1}=3.79 \mathrm{~m}_{\odot}$, $\mathrm{m}_{2}=1.67 \mathrm{~m}_{\odot}$. Moreover, the primary component is a spectroscopic binary with a velocity range of about $38 \mathrm{~km} \mathrm{~s}^{-1}$ (Oort 1975), and this triple system is therefore of the hierarchical type. From the present cluster parameters and a nuclear age of $8 \times 10^{\mathrm{s}}$ yrs, the dynamical age of the Hyades is about 40 crossing times. During its past history the cluster may have lost half of its members (Pels et al. 1975) and there would have been time for significant binary evolution.

In order to assess the dynamical relevance of the central binary, we assume a constant mean mass $\overline{\mathbf{m}}=0.7 \mathrm{~m}_{\odot}$ and scale factor $\overline{\mathrm{R}}=3 \mathrm{pc}$, as derived by Pels et al. Equation (4) then gives $\lambda \simeq 0.39$ for an initial membership of $\mathrm{N}=1000$ stars at the adopted distance of $40 \mathrm{pc}$. This result is consistent with the dynamical calculations of star cluster models if we assume that the present binary components have been among the most massive stars during a substantial part of the elapsed time. We also note that the current critical semi-major axis estimated by Equation ( 6 ) exceeds the actual value by a factor of 2 if $\bar{R}=3 \mathrm{pc}$ and $N=400$, indicating that a dynamical binary should have completed its cvolution some time ago. However, it should be emphasized that the case of dynamical formation followed by evolution cannot be distinguished from an initial binary which experiences a significant shrinkage. As for the other close visual binaries, a common origin by independent condensations seems most likely since dynamical mechanisms would involve strong recoil effects. Finally we note that possible candidates of dynamically important binaries have also been suggested for several multiple systems (van Albada 1968b); hopefully the present meeting will stimulate further interest in this fascinating subject. 


\section{REFERENCES}

Aarseth, S. J. 1971, Ap. and Space Sci., 13, 324.

Aarseth, S. J. 1973, in Vistas in Astronomy, ed. A. Beer (Oxford: Pergamon Press), 15, 13.

Aarseth, S. J. 1974, Astron. and Astrophys., 35, 237.

Aarseth, S. J. 1975, in Dynamics of Stellar Systems; ed. A. Hayli (Dordrecht: D. Reidel), 57.

Aarseth, S. J., and Heggie. D. C. 1976, Astron. and Astrophys., 53, 259.

Aarseth, S. J., and Hills, J. G. 1972, Astron. and Astrophys., 21, 225.

Agekyan, T. A., and Anosova, Z. P. 1968a, Soviet Astr.A.J., 11, 1006.

Agekyan, T. A., and Anosova, Z. P. 1968b, Astrofizika, 4,31 .

Agekyan, T. A., and Anosova, Z. P. 1971, Soviet Astr.-A.J., $15,411$.

Aitken, R. G. 1932, New General Catalogue of Double Stars, Carnegie Institution of Washington, Pub. No 417 (Washington, D.C.).

Allen, C., and Poveda, A. 1974, Proc. IAU Symposium $\mathrm{N}^{\circ} 62$, The Stability of the Solar System and of Small Stellar Systems, ed. Y. Kozai (Dordrecht: D. Reidel), 239.

Anosova, Z. P. 1969, Astrofizika, 5, 161.

Harrington, R. S. 1972, Celes. Mech., 6, 322.

Harrington, R. S. 1974, Celes. Mech., 9, 465.

Hayli, A. 1971, Ap and Space Sci., 13, 309.
Heggie, D. C. 1975, M.N.R.A.S., 173, 729.

Heggie, D. C. 1977, IAU Colloquium Iv 33, Rev. Mex. Astron. Astrof., 3, 169.

Hills, J. G. 1975, A.J., 80, 809.

Hills, J. G. 1976, M.N.R.A.S., 175, 1 P.

King, I. R. 1977 IAU Colloquium No 33 Rev. Mex. Astron. Astrof., 3, 167.

Miller, R. H. 1975, in Dynamics of Stellar Systems, ed. A. Hayli, (Dordrecht: D. Reidel), 95.

Oort, J. H. 1975, private communication.

Pels, G., Oort, J. H., and Pels-Kluyver, H. A. 1975, Astron. and Astrophys., 43, 423.

Poveda, A., Ruiz, J., and Allen, C. 1967, Bol. Obs. Tonantzintla y Tacubaya, 4, 86.

Standish, E. M. 1972, Astron. and Astrophys., 21, 185.

Szebehely, V. 1972, Celes. Mech., 6, 84.

Szebehely, V. 1974, A.J., 79, 981.

Szebehely, V. 1977, IAU Coloquium No 33, Rev. Mex. Astron. Astrof., 3, 145.

Valtonen, M. J. 1974, Proc. IAU Symposium No 62, The Stability of the Solar System and of Small Stellar Systems, ed. Y. Kozai (Dordrecht: D. Reidel), 211.

van Albada, T. S. 1968a, Bull. Astr. Inst. Netherl., 19, 479.

van Albada, T, S. 1968b, Bull. Astr. Inst. Netherl., 20, 57.

Wielen, R. 1974, in Proceedings of the First European Astronomical Meeting, ed. L. N. Mavridis (Heidelberg: Springer-Verlag), 2, 326. 